\title{
Role of type I interferon signaling and microglia in the abnormal long term potentiation and object place recognition deficits of male mice with a mutation of the Tuberous Sclerosis
} 2 gene

Manuel F. López-Aranda ${ }^{1}$, Gayle M. Boxx ${ }^{2}$, Miranda Phan ${ }^{1}$, Karen Bach ${ }^{1}$, Rochelle Mandanas ${ }^{1}$, Isaiah Herrera ${ }^{1}$, Sunrae Taloma ${ }^{1}$, Chirag Thadani ${ }^{1}$, Odilia $\mathrm{Lu}^{1}$, Raymond Bui ${ }^{1}$, Shuhan $\mathrm{Liu}^{3}$, Nan $\mathrm{Li}^{3}$, Yu Zhou ${ }^{3,4}$, Genhong Cheng ${ }^{2}$, Alcino J. Silva ${ }^{1 *}$

${ }^{1}$ Departments of Neurobiology, Psychology, Psychiatry, Integrative Center for Learning and Memory and Brain Research Institute, University of California Los Angeles, Los Angeles, CA, USA.

${ }^{2}$ Department of Microbiology, Immunology and Molecular Genetics, University of California Los Angeles, Los Angeles, CA, USA.

${ }^{3}$ Department of Physiology and Pathophysiology, School of Basic Medical Sciences, Qingdao University, Qingdao, 266071, Shandong, China.

${ }^{4}$ Institute of Brain Function and Diseases, Qingdao University, Qingdao, 266071, Shandong, China.

\begin{abstract}
Tuberous Sclerosis Complex (TSC) is a genetic disorder associated with high rates of intellectual disability and autism. Although previous studies focused on the role of neuronal deficits in the memory phenotypes of rodent models of TSC, the results presented here demonstrate a role for microglia in these deficits. Mice with a heterozygous null mutation of the Tsc 2 gene $\left(\mathrm{Tsc}^{+/-}\right)$, show deficits in hippocampal dependent tasks, as well as abnormal long-term potentiation (LTP) in the hippocampal CA1 region. Here, we show that microglia and type I interferon signaling (IFN1) have a key role in the object place recognition (OPR; a hippocampal dependent task) deficits and abnormal LTP of Tsc $2^{+/-}$male mice. Unexpectedly, we demonstrate that male, but not female, Tsc $2^{+-}$mice showed OPR deficits. Importantly, these deficits can be rescued by depletion of microglia, as well as by a genetic manipulation of a signaling pathway known to modulate microglia function (interferon-alpha/beta receptor alpha chain null mutation).
\end{abstract}


In addition to rescuing the OPR deficits, depletion of microglia also reversed the abnormal LTP of the $\mathrm{Tsc}^{+/-}$mice. Altogether, our results suggest that altered IFN1 signaling in microglia cause the abnormal LTP and OPR deficits of male $\mathrm{Tsc} 2^{+/-}$mice.

\section{Introduction}

Tuberous Sclerosis (TSC) is a genetic condition caused by mutations in either Tsc1 (1) or Tsc2 (2) genes. Autism Spectrum Disorder (ASD) (3-6), and cognitive deficits (7-9) are common in TSC. Prior studies have suggested that humans $(8,10)$, as well as Tsc1 $(7,11)$ and Tsc2 $(9,12)$ rodent models of TSC, show hippocampal dependent spatial memory deficits, as assessed in the Morris water maze $(7,9,12)$ and radial arm maze (9). In addition, other forms of hippocampal dependent memory, including contextual fear memory $(7,9,11,12)$, are impaired in TSC mouse models. Previous studies suggested that abnormal hippocampal long-term potentiation (LTP) may underlie these memory deficits $(9,13)$, thus suggesting that neuronal deficits have a principal role in the memory phenotypes of rodent models of TSC $(14,15)$.

The Object Place Recognition (OPR) task is designed to assess spatial memory and discrimination. This test is based on rodent's tendency to spend more time investigating a familiar object that has been moved to a new location $(16,17)$. Previous studies showed that impairing hippocampal function with lesions $(16,18)$, pharmacological antagonists (19), and genetic manipulations (20) resulted in impairments in OPR, demonstrating a role for the hippocampus in this task $(16,18-21)$. Here, we show that male, but not female, Tsc2 ${ }^{+/-}$mice have profound OPR deficits, that can be reversed by depletion of microglia, as well as by an interferon-alpha/beta receptor alpha chain null mutation. In addition to rescuing the OPR deficits, depletion of microglia also rescued the abnormal LTP of the $\mathrm{Tsc} 2^{+/-}$mice, a result that demonstrates a previously unrecognized role of this cell type in the spatial and LTP abnormalities of animal models of TSC.

\section{Results}

Male, but not female Tsc2 ${ }^{+/-}$mice show deficits in OPR. 
Male and female $\mathrm{Tsc} 2^{+/-}$mice and their wild type littermates (WT mice) were tested as adults (4-6 months old) in the OPR task (Fig.1A). Only male Tsc2 ${ }^{+/}$, but not female Tsc2 ${ }^{+/}$or WT mice, showed OPR deficits (no preference for the object in the novel location vs the object in the same location as during training; Fig.1B). Interestingly, all groups tested (fig.S1A) showed no deficits and equivalent performance in the object recognition memory task (NOR) (22) (fig.S1B), indicating that the OPR deficits of male $\mathrm{Tsc}^{+/-}$mice are not due to deficits in objects recognition. Recently, our laboratory has shown that early post-natal immune activation in $\mathrm{Tsc}^{+/-}$mice leads to social phenotypes (23). To determine whether immune activation can reveal OPR deficits in female $\mathrm{Tsc}^{+/-}$mice, male and female $\mathrm{Tsc}^{+/-}$mice were injected with either polyinosinic:polycytidylic acid (Poly I:C; $20 \mathrm{mg} / \mathrm{kg}$ ), or Saline (control) i.p. at post-natal day 3 (P3), P7 and P14 (fig.S2A). We then tested the mice as adults (4-6 months old) in the OPR task. Only male Tsc2 ${ }^{+-}$mice injected with Poly I:C early post-natally (Tsc2 ${ }^{+/}$Ep) or saline, but not female $\mathrm{Tsc}^{+/-}$mice, showed OPR deficits (fig.S2B). These data demonstrated that early post-natal immune activation did not reveal OPR deficits in female $\mathrm{Tsc} 2^{+/-}$mice.

\section{A single adult treatment with a mTOR inhibitor (rapamycin) can temporarily rescue the OPR deficits of male $\mathrm{Tsc}^{+/-}$mice.}

The Tsc2 gene is known to regulate mTOR signaling (24-27). Accordingly, reductions of Tsc2 cause upregulation of mTOR signaling in rodents and humans $(28,29)$. Previous results showed that an mTOR inhibitor (rapamycin) was capable of reversing multiple phenotypes observed in $\mathrm{Tsc}^{+/-}$mice (30-32). To test whether the inhibition of mTOR signaling in adult male Tsc $2^{+/-}$mice can reverse their OPR deficits, we treated those mice with rapamycin $(5 \mathrm{mg} / \mathrm{kg}$ ) or vehicle (DMSO) as adults (Fig.2A) for 5 days. Before treatment with rapamycin, the $\mathrm{Tsc} 2^{+/-}$mice showed deficits in OPR (Fig.2B), while Tsc2 ${ }^{+/-}$mice treated with rapamycin show normal OPR (preference for the object in the novel location) (Fig.2B). However, 2 months after the Rapamycin treatment, Tsc $2^{+/-}$ mice show again OPR deficits (Fig.2B). These results demonstrate the critical role of mTOR signaling in the OPR deficits of adult $\mathrm{Tsc}^{+/-}$male mice, and show that a 5-day treatment of rapamycin can only temporarily rescue the OPR deficits of $\mathrm{Tsc}^{+/-}$male mice.

Adult depletion of microglia results in the permanent rescue of the OPR deficits of male Tsc2 $^{+/-}$mice. 
Previous results from our laboratory (33) showed that microglia play a critical role in the social memory deficits of $\mathrm{Tsc} 2^{+/-}$mice with early post-natal immune activation. Therefore, we tested whether depletion of microglia could also reverse the OPR deficits of $\mathrm{Tsc} 2^{+/-}$male mice. Inhibition of the Colony Stimulating Factor 1 Receptor (CSF1R) leads to the depletion of $~ 99 \%$ of all microglia brain-wide (34) (Fig.3B). Thus, we treated male $\mathrm{Tsc}^{+/-}$mice with a CSF1R inhibitor (PLX5622 chow) or vehicle control chow (Fig.3A) for 21 days, and then tested them for OPR. Treatment with PLX5622 rescued the OPR deficits of male Tsc2 ${ }^{+/-}$mice (Fig.3C). Remarkably, we also found that this treatment results in a permanent rescue of OPR in the male $\mathrm{Tsc} 2^{+/-}$mice since these the mice showed normal OPR when they were tested 2 months after microglia depletion (Fig.3D). Histological studies demonstrated that at this time microglia had already repopulated the brain of these mice (fig 3B). These results demonstrate a role for microglia in the OPR deficits of male $\mathrm{Tsc}^{+/-}$mice.

As we mentioned above, our laboratory has previously shown that early post-natal immune activation reveals social memory deficits in $\mathrm{Tsc} 2^{+/-}$mice $\left(\mathrm{Tsc} 2^{+/-} \mathrm{Ep}\right)(33)$. Thus, we determined whether the depletion of microglia is still capable of rescuing the OPR deficits of Tsc $2^{+/-}$Ep adult mice (4-6 months old). Remarkably, treatment with PLX5622 also rescued the OPR deficits of male Tsc $2^{+/-}$Ep mice (fig.S3B), and this rescue persisted for at least 2 months (fig.S3C).

\section{The $\mathrm{Tsc}^{+/-}$mutation restricted to microglia during development, but not in adults, results in OPR deficits}

To test whether the $\mathrm{Tsc} 2^{+/-}$mutation restricted to the microglia (35) is sufficient to cause OPR deficits, we used a mouse model with a floxed Tsc2 allele specifically deleted in microglia by a Cre recombinase expressed from a microglia specific promoter $\left(\mathrm{Cx} 3 \mathrm{cr} 1\right.$; $\left.\mathrm{Cx} 3 \mathrm{cr}^{\mathrm{Cre}}-\mathrm{Tsc} 2^{\mathrm{Flox}}\right)(35)$. We tested those mice as adults (4-6 months old) for OPR (Fig.4A). Similar to germ line Tsc $2^{+/}$ male mice, male $\mathrm{Cx} 3 \mathrm{cr} 1^{\mathrm{Cre}}$ - Tsc $2^{\text {Flox }}$, but not control mice, showed OPR deficits (Fig.4B).

The results presented above (Fig.3D and fig.S3C), showed that depletion of microglia permanently recue the OPR deficits of male $\mathrm{Tsc} 2^{+/}$mice. So, once the microglia is depleted in adult mice, the $\mathrm{Tsc}^{+/-}$mutant microglia that repopulate the adult brain of these mice (after PLX treatment in adults) is not able to trigger OPR deficits. This suggests that the Tsc2 mutation, specifically during development is critical for the OPR deficits of male $\operatorname{Tsc} 2^{+/-}$mice. To test this hypothesis, we used mice with a tamoxifen-inducible $\mathrm{Tsc} 2^{+/-}$mutation restricted to microglia (the 
Cx3cr1 $1^{\text {CreER}}-$ Tsc2 $2^{\text {Flox }}$ mouse model) (36), and that could be specifically induced in adults. Thus, we activated the Tsc2 mutation in the microglia by administering 4-Hydroxytamoxifen in adult male Cx3cr1 ${ }^{\text {CreER }}$-Tsc $2^{\text {Flox }}$ mice (Fig.4C). Then, we tested those mice for OPR. Remarkably, the adult induction of the $\mathrm{Tsc} 2^{+/-}$mutation in microglia failed to induce OPR deficits in male $\mathrm{Cx} 3 \mathrm{cr} 1^{\text {CreER}}-\mathrm{Tsc}_{2}{ }^{\mathrm{Flox}}$ mice (Fig.4D), contrary to what happened when the Tsc2 ${ }^{+/-}$mutation was induced in microglia during development, a result that suggests that the $\mathrm{Tsc} 2^{+/-}$mutation affects microglia during development.

\section{Microglia depletion reverses the abnormal LTP of male $\mathrm{Tsc2}^{+/-}$mice.}

Previous studies suggested that abnormal hippocampal CA1 LTP may underlie the spatial memory deficits of $\mathrm{Tsc}^{+/-}$mice $(9,13)$. The results presented above demonstrated that microglia play a critical role in the OPR deficits of male Tsc $2^{+/-}$mice (Fig.3C and 3D; fig.S3B and S3C). To test if microglia is also responsible of the abnormal LTP of $\mathrm{Tsc}^{+/-}$mice, we treated adult male $\mathrm{Tsc}^{+/-}$Ep mice with PLX5622 or control chow and four months later, we tested their LTP (Fig.5A). We tested Tsc2 ${ }^{+/-}$Ep mice, and not $\mathrm{Tsc}^{+/-}$mice, because of the hippocampal memory deficits of the $\mathrm{Tsc}^{+/-}$Ep mice (they also include social memory deficits) are more severe than those of the $\mathrm{Tsc}^{+/-}$mice (23). Remarkably, the results show that the depletion of microglia rescued the abnormal LTP of male Tsc2 ${ }^{+/}$Ep mice (Fig.5B-5D). These results demonstrate that microglia have a critical role in the abnormal LTP of Tsc $2^{+/-}$mice, and suggest that this may contribute to their spatial learning and memory deficits.

\section{Normal short-term memory for OPR in Tsc2 ${ }^{+/-}$male mice}

Prior results (9), as well as the LTP experiments described above (Fig.5B-5D) revealed that Tsc2 $2^{+/}$ mice (with or without early-post-natal immune activation) have normal LTP tested 60 minutes post-induction, although LTP tested 3 hours post-induction, was abnormal. In the experiments described above (Fig.1), OPR memory was tested 24-hours after training. Additionally, TSC2 affects mTOR signaling $(28,29)$, and this signaling pathway has been implicated in memory consolidation (37). Thus, we next tested whether adult $\mathrm{Tsc}^{+/-}$male mice have deficits in OPR at a time both prior to memory consolidation (60 min after OPR training), and when their LTP is normal (Fig.5B-5D). Consistent with the idea that abnormal LTP may contribute to the OPR memory deficits of the $\mathrm{Tsc} 2^{+/-}$male mice, we found that these mutant mice show normal OPR 
when tested 60 min after training (Fig.6B). When tested 24 hours after training, the Tsc $2^{+/-}$male mice once again revealed OPR deficits (Fig.6B). These results support the hypothesis that the abnormal LTP of the Tsc2 ${ }^{+/-}$mice contributes to their OPR memory deficits.

\section{The interferon-alpha/beta receptor alpha chain KO (IFNAR1 $\left.{ }^{-/}\right)$prevents the OPR deficits in male $\mathrm{Tsc2}^{+/-}$mice.}

In our previous work, we determined that type I-IFN plays a critical role in the social memory deficits of male $\mathrm{Tsc}^{+/-}$mice (33). Here, we determined whether type I-IFN signaling also has a role on the OPR deficits of male Tsc $2^{+/-}$mice (Fig.7A). Remarkably, although male Tsc2 ${ }^{+/-}$mice showed robust OPR deficits (Fig.7B; as we observed in Fig.1B), male Tsc2 ${ }^{+/-}$mice with a null mutation for the interferon-alpha/beta receptor alpha chain gene ( $\left.\operatorname{Tsc} 2^{+/-} / \mathrm{IFNAR}^{-/-}\right)$showed normal OPR (Fig.7B). These results demonstrate the role of type I IFN signaling in the OPR deficits of male $\mathrm{Tsc} 2^{+/-}$mice.

\section{Discussion}

Although previous studies focused on the role of neuronal deficits in the memory phenotypes of rodent models of TSC $(14,15)$, the results presented here demonstrate a role for microglia in these memory deficits. In addition to rescuing their OPR deficits, we show that depletion of microglia also reversed the abnormal LTP of the Tsc $2^{+/-}$mice.

TSC-associated neuropsychiatric disorders, include autism, intellectual disability, and a range of other psychiatric and behavioral symptoms $(3-9,38)$. Additionally, epilepsy affects up to $80 \%$ of TSC patients, with severe seizures that can be treatment resistant, and that could contribute to their behavioral abnormalities (39). While neuronal mechanisms have played a key role in explanations of autism and intellectual disability phenotypes of TSC patients $(14,15)$, glia have been increasingly recognized to play important roles in TSC (40). For example, there is growing evidence for the role of astrocytes, microglia, and oligodendrocytes in the pathophysiology of epilepsy in TSC (40). Our previous results (33), including those presented here, also suggest that glial cells, particularly microglia, have a role in the cognitive phenotypes of TSC, and that targeting glial mechanisms could be a viable therapeutic approach for addressing their cognitive deficits. 
Pathology studies of tubers dissected from TSC epileptic patients revealed evidence of microglial activation $(41,42)$. However, seizures are common in TSC patients, and themselves may cause microglia activation (43), thus making it difficult to determine whether microglia activation is a consequence of other pathological causes in TSC, or a contributing cause for TSC phenotypes. For example, previous studies showed that mice with an astrocyte specific Tsc1 mutation showed evidence of microglia activation, as well as increased microglia size and number (44). The results presented here make a strong case that abnormalities in microglia may not simply be a consequence of changes in other cell types involved in the pathophysiology of TSC, such as astrocytes and neurons, since we showed that the $\mathrm{Tsc} 2^{+/-}$mutation restricted to microglia recreated the hippocampal memory deficits associated with animal models of $\operatorname{TSC}(7,23,45-47)$. Additionally, we also showed that the hippocampal CA1 LTP abnormalities and OPR deficits of the $\mathrm{Tsc}^{+/-}$mice could be reversed by a manipulation that depleted most microglia from the brain of these mice. Importantly, we demonstrated that a null mutation (IFNAR $1^{-/-}$) of a key signaling pathway in microglia (48) also prevented the hippocampal-dependent memory deficits of these mice. Importantly, previous RNA sequencing studies in ASD patients (49) pointed to a lasting upregulation of microglia and interferon response pathways, suggesting that our findings with an animal model of TSC may be more generally significant.

All together the results presented here demonstrate that microglia and type I interferon signaling have a key role in the electrophysiological and memory phenotypes of an animal model of TSC, a result consistent with the hypothesis that abnormal type I-IFN signaling in microglia is responsible for abnormal LTP and for hippocampal memory deficits in $\mathrm{Tsc} 2^{+/-}$mice. Importantly, these phenotypes can be reversed by depletion of microglia, a result that suggests that therapeutical strategies targeting this cell type may be a viable strategy to address cognitive impairments in TSC. 


\section{Materials and Methods}

The Chancellor's Animal Research Committee at the University of California, Los Angeles, approved the research protocols used here.

\section{Experimental Design and subject details.}

1) Tsc2 $^{+/-}$mice. We first crossed male Tsc $2^{+/-}$mice(28) with female wild type (WT) mice. Tsc2 $2^{+/-}$ male breeders were on a C57BL/6Ncrl genetic background (Charles River Laboratories, Cat.\#: 027). We used C57BL/6J females (JAX, Cat.\#: 000664) to generate experimental mice. Pregnant females were single housed and left undisturbed except for weekly cage changes. Pregnancy was determined by checking for abdominal distension. For all of the mice studied here, pregnant females were checked every day to determine the exact day when the pups were born (designated P0). Tail biopsies for genotyping were taken around P40.

2) IFNAR1 ${ }^{-/-} / /$Tsc2 $^{+/-}$mice. To test the importance of type I interferon $\alpha$ and $\beta($ IFN $\alpha$, $\beta$ ) for the phenotype we observed in male $\mathrm{Tsc} 2^{+/-}$mice, we generated mice that included both the $\mathrm{Tsc} 2^{+/-}$and

IFNAR $1^{-/-}$null mutations by crossing $\mathrm{Tsc}^{+/-}$male breeders with IFNAR $1^{-/-}$females (bred on a C57Bl/6J background) followed by intercrosses. Tail biopsies for genotyping were taken around P40.

3) Cx3cr1 $1^{\text {Cre }}$-Tsc2 ${ }^{\text {Flox }}$ mice. To confirm whether microglia play a critical role in the hippocampaldependent memory deficits that male $\mathrm{Tsc} 2^{+/-}$mice show, we crossed $\mathrm{Cx} 3 \mathrm{cr} 1^{\text {Cre }}$ mice (JAX stock $\# 025524)$ with Tsc $2^{\text {Flox }}$ mice (JAX stock \#027458) to generate mice that carry the Tsc2 mutation only in microglia. Tail biopsies for genotyping were taken around P40.

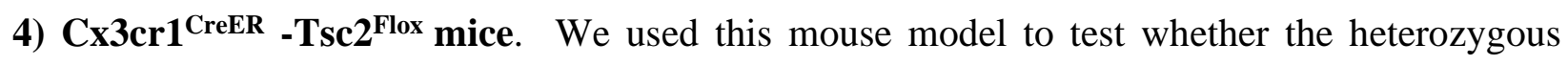
deletion of the Tsc2 gene specifically in adult microglia can trigger spatial memory deficits in adult male mice. We also used those mice to determine if this Tsc2 gene deletion in adult microglia can create a window of vulnerability where a post-natal immune activation could trigger spatial memory deficits in female $\mathrm{Tsc}^{+/-}$mice. We crossed $\mathrm{Cx} 3 \mathrm{Cr} 1^{\mathrm{CreER}}$ mice expressing tamoxifeninducible Cre recombinase (JAX stock \# 021160) with Tsc $2^{\text {Flox }}$ mice (JAX stock \#027458) to 
generate mice that carry the $\mathrm{Tsc} 2^{+/-}$mutation only in microglia of adults. The experimental mice were injected intraperitoneally with 4-Hydroxytamoxifen (4-OHT) (Sigma Aldrich; Cat.\#:H6278$50 \mathrm{mg} ; 75 \mathrm{mg} / \mathrm{kg}$ ) once per day during 4 consecutive days. Tail biopsies for genotyping were taken around $\mathrm{P} 40$.

\section{Method details.}

\section{1) Object Place Recognition test (OPR).}

Prior to the test, mice were handled for eight minutes daily for 6 consecutive days. Then, in the next two days, they were habituated in the OPR open field $(41.5 \times 41.5 \times 40.5 \mathrm{~cm}) 2$ days for 12 minutes. During the training session, mice were placed back in the OPR open-field, this time with 2 identical objects, and they were allowed to explore freely for 7 minutes. After either a 1 hour or a $24 \mathrm{~h}$ interval, mice were tested for OPR with the previously presented objects, one of them in a new location. The object on the new location during test was counterbalanced between trials. The open-field was cleaned after each session. Sessions were video recorded and scored offline by either one or two observers, as described above. The variation between observers was normally less than 2 seconds in the 7 minutes exploration sessions. Exploration was counted only when the test mouse touched with its nose one of the objects. All experiments and scoring were carried out blind to genotype and treatment condition. The objects included in this study were small bottles or containers of different shapes.

\section{2) Novel Object Recognition test (NOR).}

The Novel Object Recognition test (NOR) was carried out as described previously (22), twentyfour hours after OPR. During the training session, mice were placed in the NOR open-field (same as the OPR open field), with 2 identical objects, and they were allowed to explore freely for 7 minutes. After a $24 \mathrm{~h}$ interval, mice were tested for object memory with the previously presented object and a new object. Location of the new object during the test was counterbalanced between trials. Mice were trained or tested once per day. The open-field was cleaned after each session. Sessions were video recorded and scored offline as described above. The variation between observers was normally less than 2 seconds in the 7 minutes exploration sessions. Exploration was counted only when the test mouse touched with its nose the objects. All experiments and scoring 
were carried out blind to genotype and treatment condition. The objects included in this study were small bottles or containers of different shapes.

\section{3) Poly I:C administration.}

Poly I:C potassium salt (Sigma; Cat.\#: P9582-50MG; Poly I:C is supplied at $10 \%$ of the total weight of the salt; dosage was based on the weight of poly I:C itself) was dissolved in vehicle solution ( $0.9 \%$ sterile saline) before use. Tsc $2^{+/-}$mice were injected intraperitoneally with Poly I:C $(20 \mathrm{mg} / \mathrm{kg})$ or vehicle at P3, P7 and P14.

\section{4) Rapamycin treatment}

Rapamycin (5 mg/kg; LC Laboratories, Cat.\#: R-5000) was freshly dissolved in vehicle solution (100\% DMSO; Sigma-Aldrich, Cat.\#: D5879-500ML) before use. Adults (4-6 months) Tsc2 ${ }^{+/}$ mice were treated with a single intraperitoneal injection of rapamycin $(5 \mathrm{mg} / \mathrm{kg})$ or vehicle (DMSO) daily for 5 days prior to the OPR test. Mice were tested for OPR $18 \mathrm{~h}$ after the last injection of rapamycin or DMSO as well as 2 months later.

\section{5) PLX5622 treatment}

PLX5622 and control rodent diet was provided by Plexxikon Inc. and formulated in AIN-76A standard chow by Research Diets. Adult (4-6 months old) Tsc $2^{+/-}$mice were fed with PLX5622 (1200mg/kg; Cat.\#: D11100404i) or control chow (Cat.\#: D10001i) for 21 days (50). At day 21 of PLX treatment, mice were tested for OPR test. Mice were tested again in the OPR test, 2 months after the last day of PLX treatment.

\section{6) 4-Hydroxytamoxifen (4-OHT) treatment}

A solution of 4-Hydroxytamoxifen was prepared using pure Ethanol (Sigma Aldrich; Cat.\#:E70231L) and corn oil (Sigma Aldrich; Cat.\#: C8267-500ML). First, 4-OHT (125mg/ml; Sigma Aldrich, Cat.\#: H6278-50mg) was added to ethanol (100\%; pure) and incubated at $55^{\circ} \mathrm{C}$ with vortexing for $15 \mathrm{~min}$. Then, corn oil was added to a final concentration of 4-OHT of $12.5 \mathrm{mg} / \mathrm{ml}$. Adult experimental mice were injected intraperitoneally with either $75 \mathrm{mg} / \mathrm{kg} 4-\mathrm{OHT}$ or vehicle once a day for 4 consecutive days. 


\section{7) Immunohistochemistry}

Immunohistochemistry was performed as described earlier (51). Briefly, mice were perfused transcardially with a fixative containing $4 \%$ paraformaldehyde, and cryoprotected with $30 \%$ sucrose. Coronal and sagittal brain sections $60 \mu \mathrm{m}$ thick were incubated overnight at $4^{\circ} \mathrm{C}$ with monoclonal rabbit anti-Iba1 (1:1000 dilutions; Wako Chemicals, Cat.\#: 019-19741). The sections were then incubated for 90 minutes with Alexa Fluor 488 goat anti-rabbit IgG (1:500 dilutions; Invitrogen, Cat.\#: A11011). After that, the sections were incubated in DAPI (1:1000 dilutions) for 15 minutes and then in PBS for 14 minutes. The immunofluorescence labeling was detected with a confocal microscope.

\section{8) Hippocampal slice physiology}

Coronal hippocampal slices (350 $\mu \mathrm{m}$ in thickness) were freshly made with a Leica VT-1000 vibratome in ice-cold cutting solution containing: $7 \mathrm{mM} \mathrm{MgSO}_{4}, 1 \mathrm{mM} \mathrm{CaCl}_{2}, 2.5 \mathrm{mM} \mathrm{KCl}, 26 \mathrm{mM}$ $\mathrm{NaHCO}_{3}, 1 \mathrm{mM} \mathrm{NaH}_{2} \mathrm{PO}_{4}, 30 \mathrm{mM}$ Glucose, $1.3 \mathrm{mM}$ sodium L-ascorbate, $1 \mathrm{mM}$ kynurenic acid, $3 \mathrm{mM}$ sodium pyruvate, and $119 \mathrm{mM}$ choline chloride. Slices were recovered in a solution, consisting of $85 \mathrm{mM} \mathrm{NaCl}, 2.5 \mathrm{mM} \mathrm{KCl}, 4 \mathrm{mM} \mathrm{MgCl}_{2}, 0.5 \mathrm{mM} \mathrm{CaCl}_{2}, 1.25 \mathrm{mM} \mathrm{NaH}_{2} \mathrm{PO}_{4}, 24$ $\mathrm{mM} \mathrm{NaHCO}_{3}, 25 \mathrm{mM}$ glucose and $50 \mathrm{mM}$ sucrose, for at least $1 \mathrm{~h}$ at room temperature. Slices were then transferred to the submerged recording chamber and continuously perfused with artificial cerebrospinal fluid (ACSF, $30{ }^{\circ} \mathrm{C}$ ) at a rate of $\sim 3 \mathrm{ml} / \mathrm{min}$. ACSF contains (in $\mathrm{mM}$ ) 120 $\mathrm{NaCl}, 3.5 \mathrm{KCl}, 1.3 \mathrm{MgCl}_{2}, 2.5 \mathrm{CaCl}_{2}, 1.25 \mathrm{NaH}_{2} \mathrm{PO}_{4}, 26 \mathrm{NaHCO}_{3}$ and 10 D-glucose. All solutions (PH 7.2 7.4, Osmo 290 310) were oxygenated with 95\% $\mathrm{O}_{2} / 5 \% \mathrm{CO}_{2}$ mixture gas.

Field excitatory postsynaptic potentials (fEPSPs) in the Schaffer collateral-CA1 (SC-CA1) in the dorsal hippocampus were evoked with a FHC bipolar platinum microelectrode (Ehninger et al., 2008). The input-output (I/O) curve was constructed by varying stimuli intensity (10 100 $\mu \mathrm{A})$, while measuring the corresponding pre-synaptic volley and the initial fEPSPs slope. Long-term potentiation (LTP) of SC-CA1 synapses was induced by a $100 \mathrm{~Hz}$ (1s) tetanus. All stimulation pulses were $100 \mu \mathrm{s}$ in duration and at 1/3 1/2 of the intensity that induces a maximal fEPSP response. Data were obtained using MultiClamp 700B amplifier equipped with a digidata 1440A interface and pCLAMP 10.0 software (Axon Instruments). Digitalized signals were sampled at 10 $\mathrm{kHz}$ and filtered at $2 \mathrm{kHz}$. Initial fEPSPs slopes after tetanic stimulation were normalized to the 
average baseline fEPSP slopes. For statistical analyses, two-way repeated measure ANOVAs and a Sidak's multiple comparisons test were used on the average of the first $5 \mathrm{~min}$ and the last $20 \mathrm{~min}$ of recording after LTP induction.

\section{Statistical Analysis}

Mouse behavioral data are presented as mean \pm SEM as well as individual data. For behavioral experiments, statistics presented in the figures were based on Student's t-tests. The data was also analyzed using two-way ANOVA plus Holm-Sidak post-hoc analyses with identical results. $\mathrm{P}<0.05$ was considered significant. $\mathrm{P}>0.05, \mathrm{P}<0.05, \mathrm{P}<0.01, \mathrm{P}<0.001$ and $\mathrm{P}<0.0001$ were denoted as n.s. (non-significant), *,**, ***, ****, respectively. GraphPad Prism 7 software was used to perform statistical analyses and for generating graphical representations of the data.

\section{Acknowledgments}

We thank, Evangelina Lugo and Kyra Dingle for their excellent contributions to the development, maintenance and treatment of the experimental mice. Thanks to the members of the Silva laboratory for discussions that contributed to experiments and analyses described here. Special thanks to Plexxikon Inc. for providing both PLX and control chow.

Funding: This work was supported by the Human Frontier Science Program (Ref.\#: LT000822/2011-L), Children's Tumor Foundation (Grant\#: 2014-01-014), Takeda Pharmaceutical Company Limited and NIH R01 MH084315 to AJS.

Author contributions: Conceptualization, A.J.S. and M.F.L.A.; Methodology, A.J.S. and M.F.L.A.; Formal Analyses, M.F.L.A., S.L., N.L. and Y.Z.; Investigation, M.F.L.A. G.M.B., M.P., K.B., R.M., I.H., S.T., C.T., O.L., R.B.; Resources: G.C., and Plexicon Inc.; Writing-Original Draft, M.F.L.A. and A.J.S.; Writing-Review \& Editing, A.J.S. and M.F.L.A.; Visualization, A.J.S. and M.F.L.A.; Supervision, A.J.S.; Funding Acquisition, A.J.S and M.F.L.A.

Competing interests: The authors declare no competing interests. 
Data and materials availability: PLX5622 was obtained under a material transfer agreement with Plexxikon. All data needed to evaluate the conclusions in the paper are present in the paper and/or the Supplementary Materials. 


\section{References}

1. M. van Slegtenhorst et al., Identification of the tuberous sclerosis gene TSC1 on chromosome 9q34. Science 277, 805-808 (1997).

2. C. European Chromosome 16 Tuberous Sclerosis, Identification and characterization of the tuberous sclerosis gene on chromosome 16. Cell 75, 1305-1315 (1993).

3. S. L. Smalley, P. E. Tanguay, M. Smith, G. Gutierrez, Autism and tuberous sclerosis. $J$ Autism Dev Disord 22, 339-355 (1992).

4. H. Cass, D. Sekaran, G. Baird, Medical investigation of children with autistic spectrum disorders. Child Care Health Dev 32, 521-533 (2006).

5. T. T. Gipson, G. Gerner, M. A. Wilson, M. E. Blue, M. V. Johnston, Potential for treatment of severe autism in tuberous sclerosis complex. World journal of clinical pediatrics 2, 16-25 (2013).

6. A. Vignoli et al., Autism spectrum disorder in tuberous sclerosis complex: searching for risk markers. Orphanet journal of rare diseases 10, 154 (2015).

7. S. M. Goorden, G. M. van Woerden, L. van der Weerd, J. P. Cheadle, Y. Elgersma, Cognitive deficits in Tsc1+/- mice in the absence of cerebral lesions and seizures. Ann Neurol 62, 648-655 (2007).

8. K. Ridler et al., Neuroanatomical correlates of memory deficits in tuberous sclerosis complex. Cereb Cortex 17, 261-271 (2007).

9. D. Ehninger et al., Reversal of learning deficits in a Tsc2+/- mouse model of tuberous sclerosis. Nat Med 14, 843-848 (2008).

10. P. J. de Vries, Targeted treatments for cognitive and neurodevelopmental disorders in tuberous sclerosis complex. Neurotherapeutics 7, 275-282 (2010).

11. N. Haji et al., Tsc1 haploinsufficiency in Nkx2.1 cells upregulates hippocampal interneuron mTORC1 activity, impairs pyramidal cell synaptic inhibition, and alters contextual fear discrimination and spatial working memory in mice. Mol Autism 11, 29 (2020).

12. D. Ehninger, A. J. Silva, Increased levels of anxiety-related behaviors in a Tsc 2 dominant negative transgenic mouse model of tuberous sclerosis. Behav Genet 41, 357-363 (2011). 
13. C. von der Brelie, R. Waltereit, L. Zhang, H. Beck, T. Kirschstein, Impaired synaptic plasticity in a rat model of tuberous sclerosis. Eur J Neurosci 23, 686-692 (2006).

14. D. Ehninger, P. J. de Vries, A. J. Silva, From mTOR to cognition: molecular and cellular mechanisms of cognitive impairments in tuberous sclerosis. J Intellect Disabil Res 53, 838-851 (2009).

15. P. E. Davis, J. M. Peters, D. A. Krueger, M. Sahin, Tuberous Sclerosis: A New Frontier in Targeted Treatment of Autism. Neurotherapeutics 12, 572-583 (2015).

16. D. G. Mumby, S. Gaskin, M. J. Glenn, T. E. Schramek, H. Lehmann, Hippocampal damage and exploratory preferences in rats: memory for objects, places, and contexts. Learn Mem 9, 49-57 (2002).

17. A. Vogel-Ciernia, M. A. Wood, Examining object location and object recognition memory in mice. Curr Protoc Neurosci 69, 831 31-17 (2014).

18. G. R. Barker, E. C. Warburton, When is the hippocampus involved in recognition memory? J Neurosci 31, 10721-10731 (2011).

19. J. Haettig et al., HDAC inhibition modulates hippocampus-dependent long-term memory for object location in a CBP-dependent manner. Learn Mem 18, 71-79 (2011).

20. R. M. Barrett et al., Hippocampal focal knockout of CBP affects specific histone modifications, long-term potentiation, and long-term memory. Neuropsychopharmacology 36, 1545-1556 (2011).

21. Y. Sun et al., CA1-projecting subiculum neurons facilitate object-place learning. Nat Neurosci 22, 1857-1870 (2019).

22. A. Ennaceur, J. Delacour, A new one-trial test for neurobiological studies of memory in rats. 1: Behavioral data. Behav Brain Res 31, 47-59 (1988).

23. Manuel F. López-Aranda et al., Post-natal immune activation causes social deficits in a mouse model of Tuberous Sclerosis: role of microglia and clinical implications. Science Advances 7, (2021).

24. D. J. Kwiatkowski, B. D. Manning, Tuberous sclerosis: a GAP at the crossroads of multiple signaling pathways. Hum Mol Genet 14 Spec No. 2, R251-258 (2005).

25. J. Huang, C. C. Dibble, M. Matsuzaki, B. D. Manning, The TSC1-TSC2 complex is required for proper activation of mTOR complex 2. Mol Cell Biol 28, 4104-4115 (2008). 
26. J. Huang, B. D. Manning, A complex interplay between Akt, TSC2 and the two mTOR complexes. Biochem Soc Trans 37, 217-222 (2009).

27. Y. Li, M. N. Corradetti, K. Inoki, K. L. Guan, TSC2: filling the GAP in the mTOR signaling pathway. Trends Biochem Sci 29, 32-38 (2004).

28. H. Onda, A. Lueck, P. W. Marks, H. B. Warren, D. J. Kwiatkowski, Tsc2(+/-) mice develop tumors in multiple sites that express gelsolin and are influenced by genetic background. J Clin Invest 104, 687-695 (1999).

29. A. S. Prabowo et al., Fetal brain lesions in tuberous sclerosis complex: TORC1 activation and inflammation. Brain pathology 23, 45-59 (2013).

30. S. H. Dan Ehninger, Carrie Shilyansky, Yu Zhou,1 Weidong Li, David J Kwiatkowski, Vijaya Ramesh, and Alcino J Silva, Reversal of learning deficits in a Tsc2+/- mouse model of tuberous sclerosis. Nature Medicine 18, 5 (2008).

31. P. T. Tsai et al., Autistic-like behaviour and cerebellar dysfunction in Purkinje cell Tsc1 mutant mice. Nature 488, 647-651 (2012).

32. A. Sato et al., Rapamycin reverses impaired social interaction in mouse models of tuberous sclerosis complex. Nature communications 3, 1292 (2012).

33. M. F. Lopez-Aranda et al., Postnatal immune activation causes social deficits in a mouse model of tuberous sclerosis: Role of microglia and clinical implications. Sci Adv 7, eabf2073 (2021).

34. M. R. Elmore et al., Colony-stimulating factor 1 receptor signaling is necessary for microglia viability, unmasking a microglia progenitor cell in the adult brain. Neuron $\mathbf{8 2}$, 380-397 (2014).

35. S. Yona et al., Fate mapping reveals origins and dynamics of monocytes and tissue macrophages under homeostasis. Immunity 38, 79-91 (2013).

36. C. N. Parkhurst et al., Microglia promote learning-dependent synapse formation through brain-derived neurotrophic factor. Cell 155, 1596-1609 (2013).

37. P. Bekinschtein et al., mTOR signaling in the hippocampus is necessary for memory formation. Neurobiol Learn Mem 87, 303-307 (2007).

38. P. J. de Vries et al., Tuberous sclerosis associated neuropsychiatric disorders (TAND) and the TAND Checklist. Pediatric neurology 52, 25-35 (2015). 
39. C. J. Chu-Shore, P. Major, S. Camposano, D. Muzykewicz, E. A. Thiele, The natural history of epilepsy in tuberous sclerosis complex. Epilepsia 51, 1236-1241 (2010).

40. M. Wong, The role of glia in epilepsy, intellectual disability, and other neurodevelopmental disorders in tuberous sclerosis complex. J Neurodev Disord 11, 30 (2019).

41. K. Boer et al., Inflammatory processes in cortical tubers and subependymal giant cell tumors of tuberous sclerosis complex. Epilepsy Res 78, 7-21 (2008).

42. K. Boer et al., Clinicopathological and immunohistochemical findings in an autopsy case of tuberous sclerosis complex. Neuropathology 28, 577-590 (2008).

43. L. A. Shapiro, L. Wang, C. E. Ribak, Rapid astrocyte and microglial activation following pilocarpine-induced seizures in rats. Epilepsia 49 Suppl 2, 33-41 (2008).

44. B. Zhang, J. Zou, L. Han, N. Rensing, M. Wong, Microglial activation during epileptogenesis in a mouse model of tuberous sclerosis complex. Epilepsia 57, 13171325 (2016).

45. D. Ehninger et al., Reversal of learning deficits in a Tsc2+/- mouse model of tuberous sclerosis. Nat Med 14, 843-848 (2008).

46. B. D. Auerbach, E. K. Osterweil, M. F. Bear, Mutations causing syndromic autism define an axis of synaptic pathophysiology. Nature 480, 63-68 (2011).

47. L. H. Zeng et al., Abnormal glutamate homeostasis and impaired synaptic plasticity and learning in a mouse model of tuberous sclerosis complex. Neurobiol Dis 28, 184-196 (2007).

48. A. McDonough, R. V. Lee, J. R. Weinstein, Microglial Interferon Signaling and White Matter. Neurochem Res 42, 2625-2638 (2017).

49. N. N. Parikshak et al., Genome-wide changes in lncRNA, splicing, and regional gene expression patterns in autism. Nature 540, 423-427 (2016).

50. N. N. Dagher et al., Colony-stimulating factor 1 receptor inhibition prevents microglial plaque association and improves cognition in 3xTg-AD mice. J Neuroinflammation 12, 139 (2015).

51. M. F. Lopez-Aranda, M. J. Acevedo, F. J. Carballo, A. Gutierrez, Z. U. Khan, Localization of the GoLoco motif carrier regulator of G-protein signalling 12 and 14 proteins in monkey and rat brain. Eur J Neurosci 23, 2971-2982 (2006). 


\section{Figures}

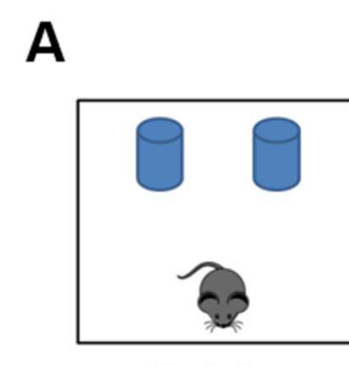

Training

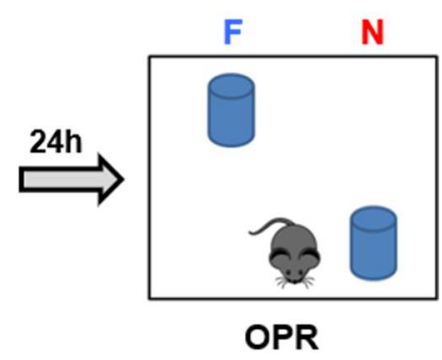

B
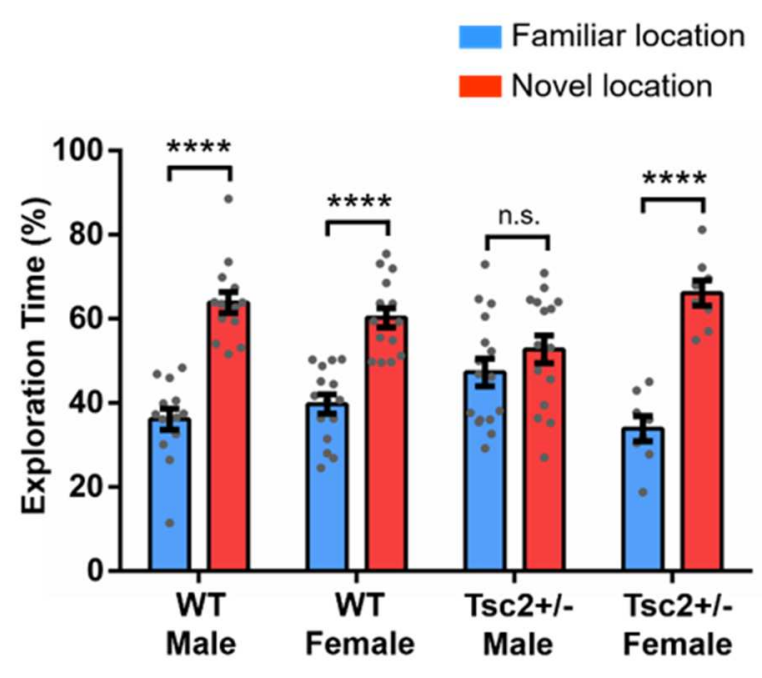

Fig. 1. Male but not female Tsc2 ${ }^{+/-}$mice show OPR deficits.

(A) OPR outline. (B) Graph shows the \% of time that mice spent actively exploring the objects in the novel and familiar locations. WT/male $(\mathrm{n}=14 ; \mathrm{P}<0.0001, \mathrm{t}=7.81)$, WT/female $(\mathrm{n}=15$; $\mathrm{P}<0.0001, \mathrm{t}=6.35)$ and $\mathrm{Tsc} 2^{+/-} /$female $(\mathrm{n}=8 ; \mathrm{P}<0.0001, \mathrm{t}=7.59)$, show normal OPR (they explore significantly more the object in the novel location than the object in the familiar location) but $\mathrm{Tsc}^{+/-} /$male $(\mathrm{n}=16 ; \mathrm{P}=0.25, \mathrm{t}=1.16)$ mice show OPR deficits (show no preference for the object in the novel location). Data represent means \pm SEM as well as individual data. 


\section{A}

\section{Rapamycin or DMSO}
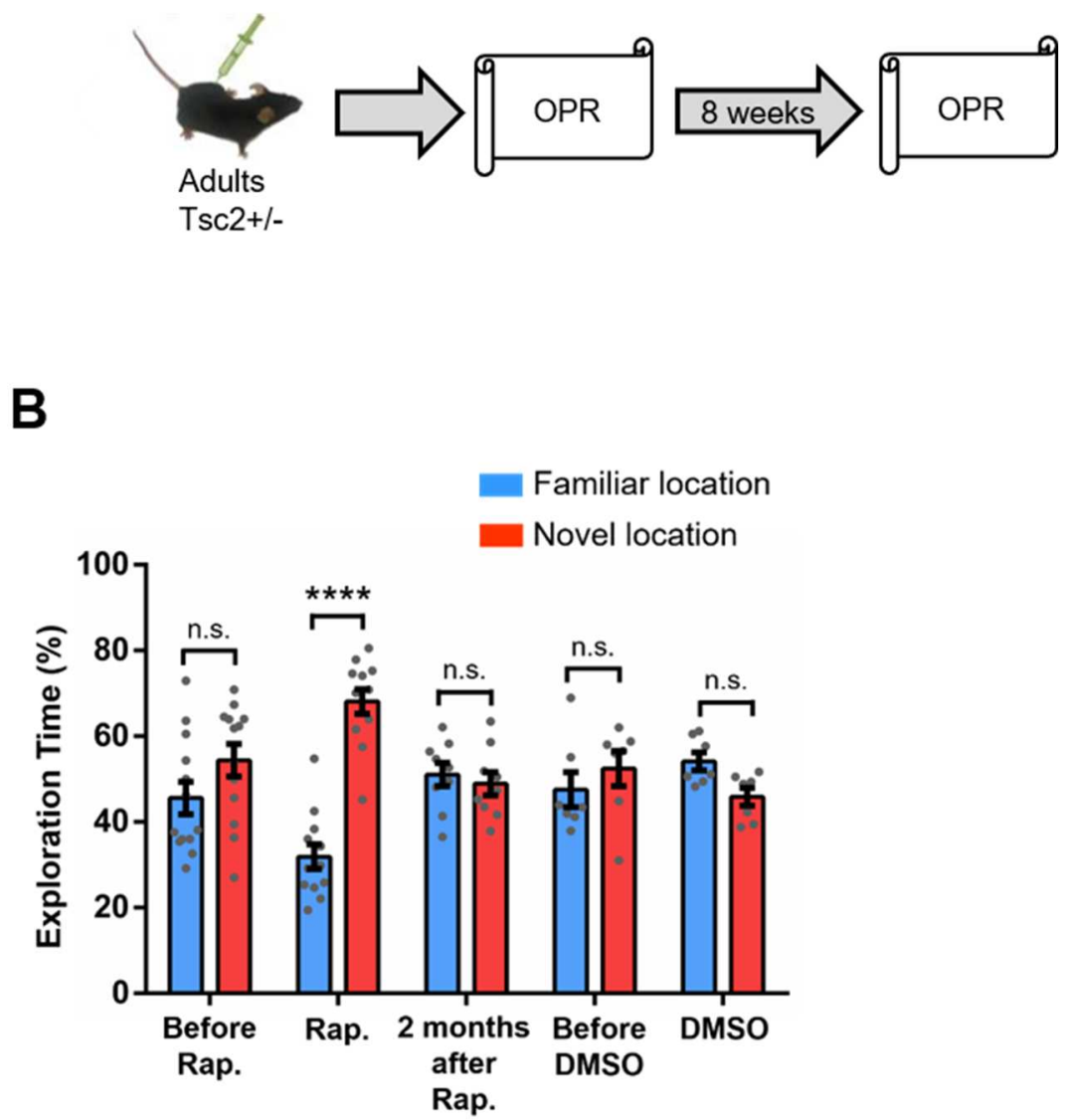

\section{Fig. 2. Effects of rapamycin on the OPR deficits of male Tsc2 ${ }^{+/-}$mice.}

(A) Outline of the treatment with rapamycin (Rap) or DMSO and behavior approach. (B) Tsc $2^{+/-}$ male mice before rapamycin $(\mathrm{n}=13 ; \mathrm{P}=0.11, \mathrm{t}=1.64)$ treatment show OPR deficits. $\mathrm{Tsc} 2^{+/-}$mice during rapamycin treatment $(\mathrm{n}=12 ; \mathrm{P}<0.0001, \mathrm{t}=8.90)$ show normal OPR. In contrast, $\mathrm{Tsc} 2^{+/-}$mice 2-months after rapamycin $(\mathrm{n}=9 ; \mathrm{P}=0.58, \mathrm{t}=0.55)$ show again OPR deficits. Data represent means \pm SEM as well as individual data points. 
A

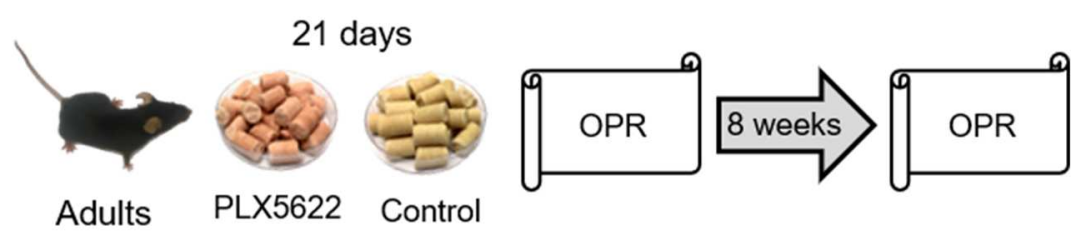

B

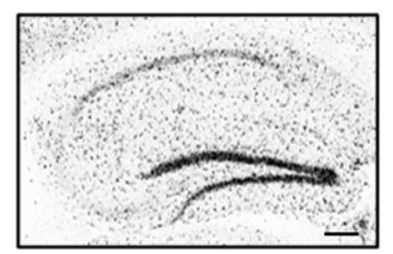

Control

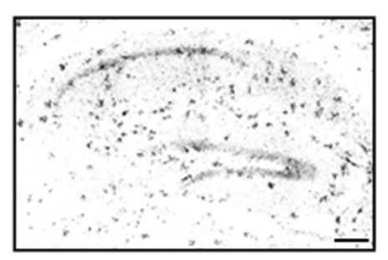

PLX5622

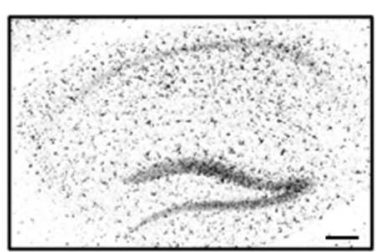

2 months after PLX5622
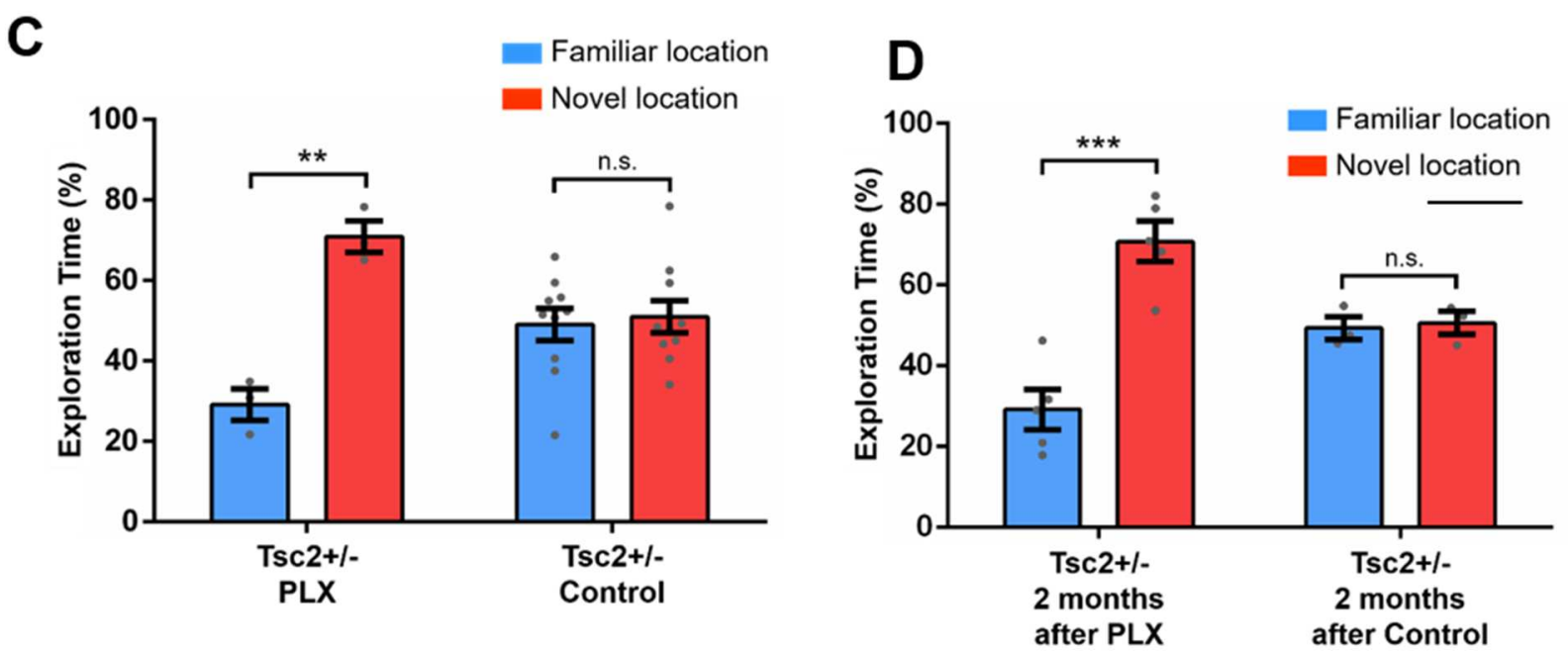

Fig. 3. Evidence for a critical role of microglia in the OPR memory deficits of male $\mathrm{Tsc2}^{+/-}$ mice.

(A) Outline of the treatment with PLX5622 (PLX; depletes microglia) or Control chow and behavior approach. (B) IBA1 immuno staining of Tsc $2^{+/-}$Control, PLX-mice and mice 2 months after PLX. The treatment with PLX5622 led to the elimination of microglia in the whole brain (Hippocampus shown as example) compared to the control group (Hippocampus shown as 
example). Two months after PLX the microglia had repopulated the brain (Hippocampus shown as example). $(\mathbf{C}) \mathrm{Tsc} 2^{+/-} / \mathrm{PLX}$ mice $(\mathrm{n}=3 ; \mathrm{P}<0.01, \mathrm{t}=7.59)$, but not Tsc2 ${ }^{+/-} /$Control $(\mathrm{n}=10 ; \mathrm{P}=0.73$, $\mathrm{t}=0.33$ ) mice, show normal OPR memory. (D) $\mathrm{Tsc} 2^{+/-}$mice two months after $\operatorname{PLX}(\mathrm{n}=5 ; \mathrm{P}<0.001$, $\mathrm{t}=5.92)$, but not $\mathrm{Tsc}^{+/-}$mice 2 months after Control $(\mathrm{n}=3 ; \mathrm{P}=0.76, \mathrm{t}=0.31)$, show normal OPR memory. Data represent mean \pm SEM as well as individual data. Bar: $200 \mu \mathrm{m}$. 
A

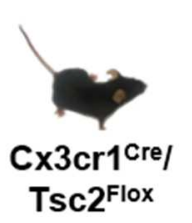

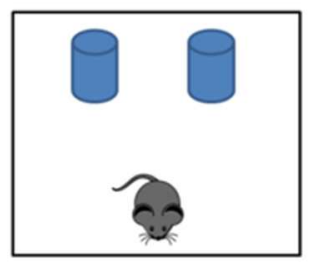

Training

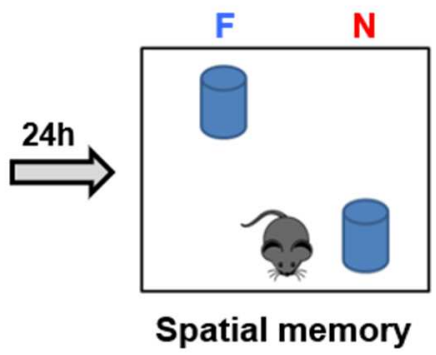

B

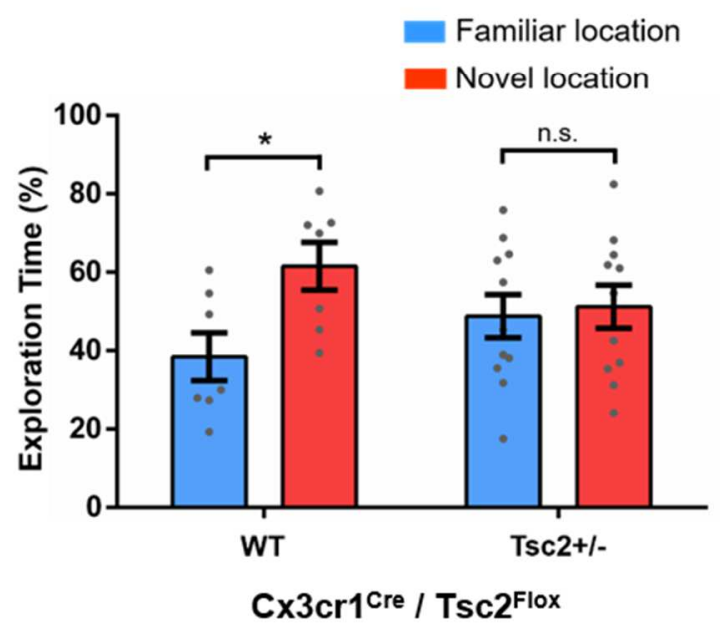

C 4-Hydroxytamoxifen (4-OHT) or Vehicle

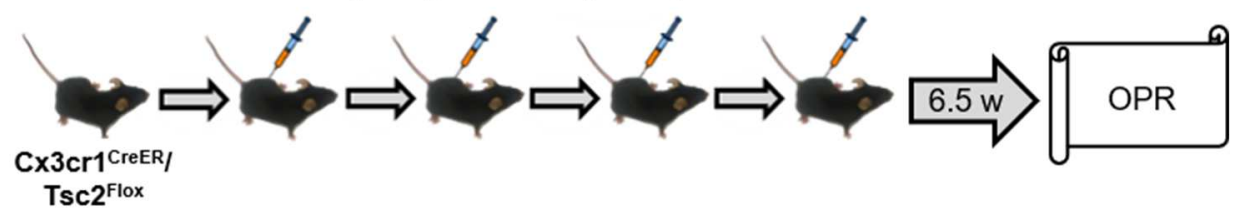

D

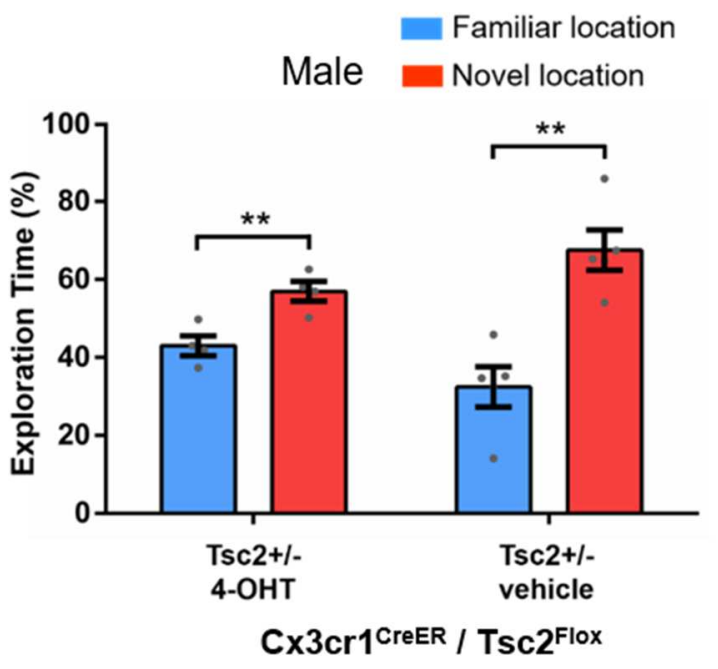


Fig. 4. The Tsc2 mutation restricted to microglia during development (but not in adults mice) leads to OPR memory deficits.

(A) Outline of Behavior approach for $\mathrm{Cx} 3 \mathrm{cr} 1^{\mathrm{Cre}} / \mathrm{Tsc} 2^{\mathrm{Flox}}$. (B) Male WT (n=7; P<0.05, t=2.69) show normal OPR. In contrast, $\mathrm{Cx} 3 \mathrm{cr} 1^{\mathrm{Cre}} / \mathrm{Tsc} 2^{\mathrm{Flox}}\left(\mathrm{Tsc} 2^{+-}\right)(\mathrm{n}=11 ; \mathrm{P}=0.76, \mathrm{t}=0.3)$ show deficits in OPR. (C) Time line for injections of 4-Hydroxyramoxifen or vehicle and behavior approach. (D) Male Cx3cr1 $1^{\text {CreER} / T s c 2 ~} 2^{\text {Flox}} /$ vehicle mice $(\mathrm{n}=5 ; \mathrm{P}<0.01, \mathrm{t}=4.8)$ and male $\mathrm{Cx} 3 \mathrm{cr} 1^{\mathrm{CreER}} / \mathrm{Tsc} 2^{\text {Flox }} / 4$ OHT mice $(\mathrm{n}=4 ; \mathrm{P}<0.01, \mathrm{t}=3.85)$, show normal OPR.

Data represent means $\pm \mathrm{SEM}$ as well as values for individual mice. As indicated, in (B), Tsc2 ${ }^{+/}$ represents $\mathrm{Cx} 3 \mathrm{cr} 1^{\mathrm{Cre}} / \mathrm{Tsc} 2^{\mathrm{Flox}}$ and in (D), Tsc2 ${ }^{+/-}$represents $\mathrm{Cx} 3 \mathrm{cr} 1^{\mathrm{CreER}} / \mathrm{Tsc} 2^{\mathrm{Flox}}$. 
A

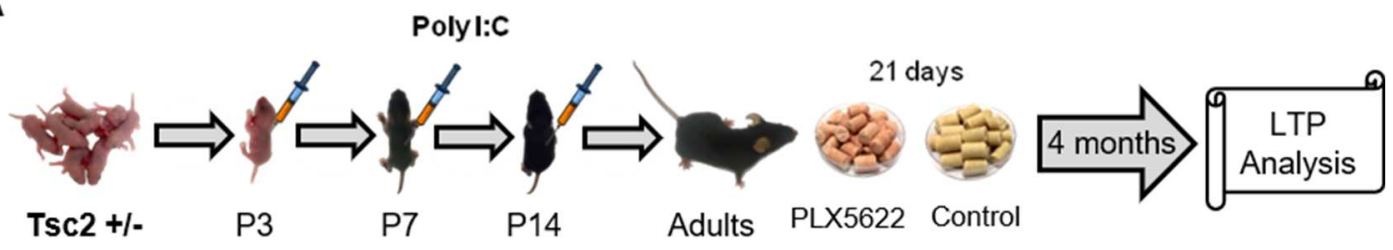

B

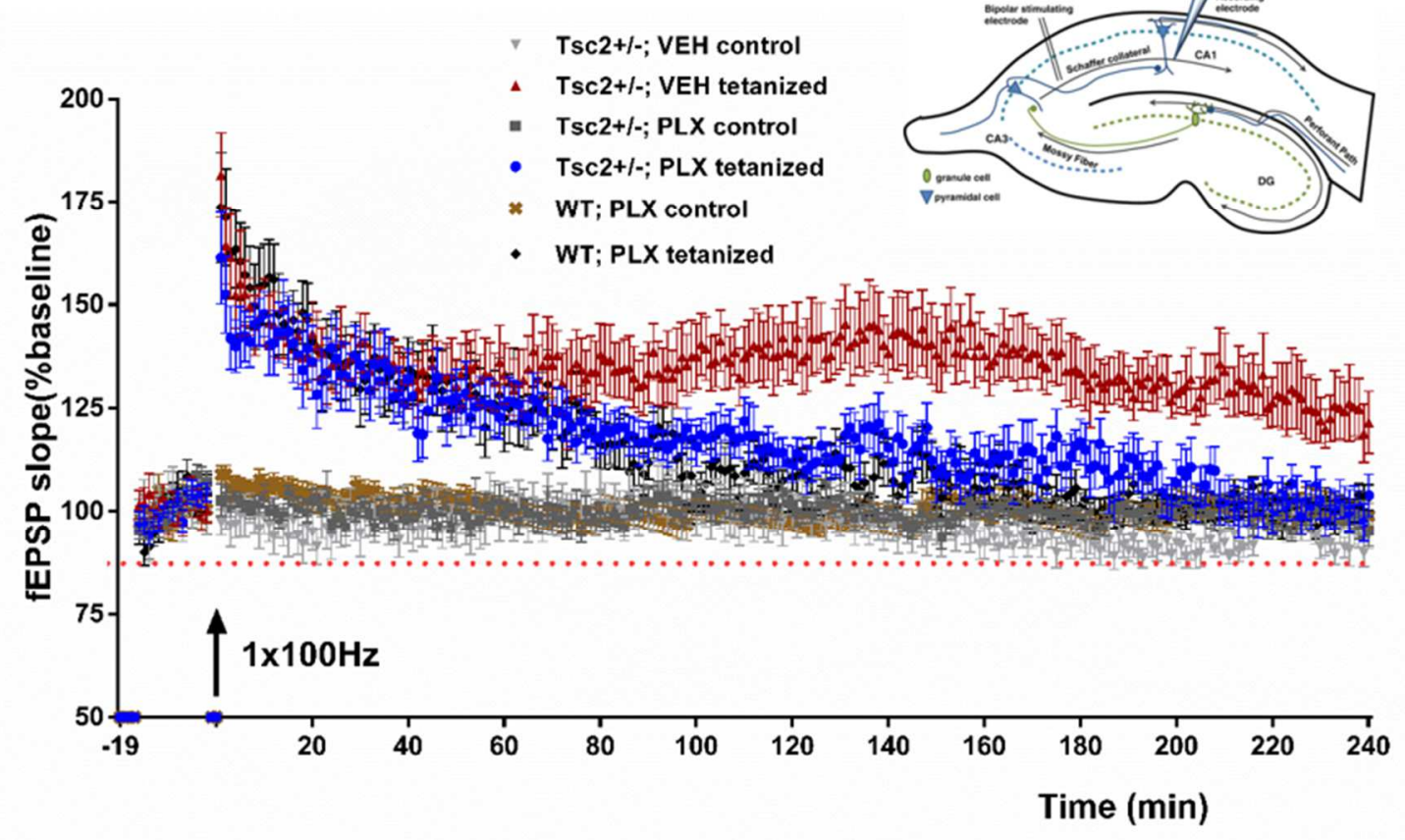

C
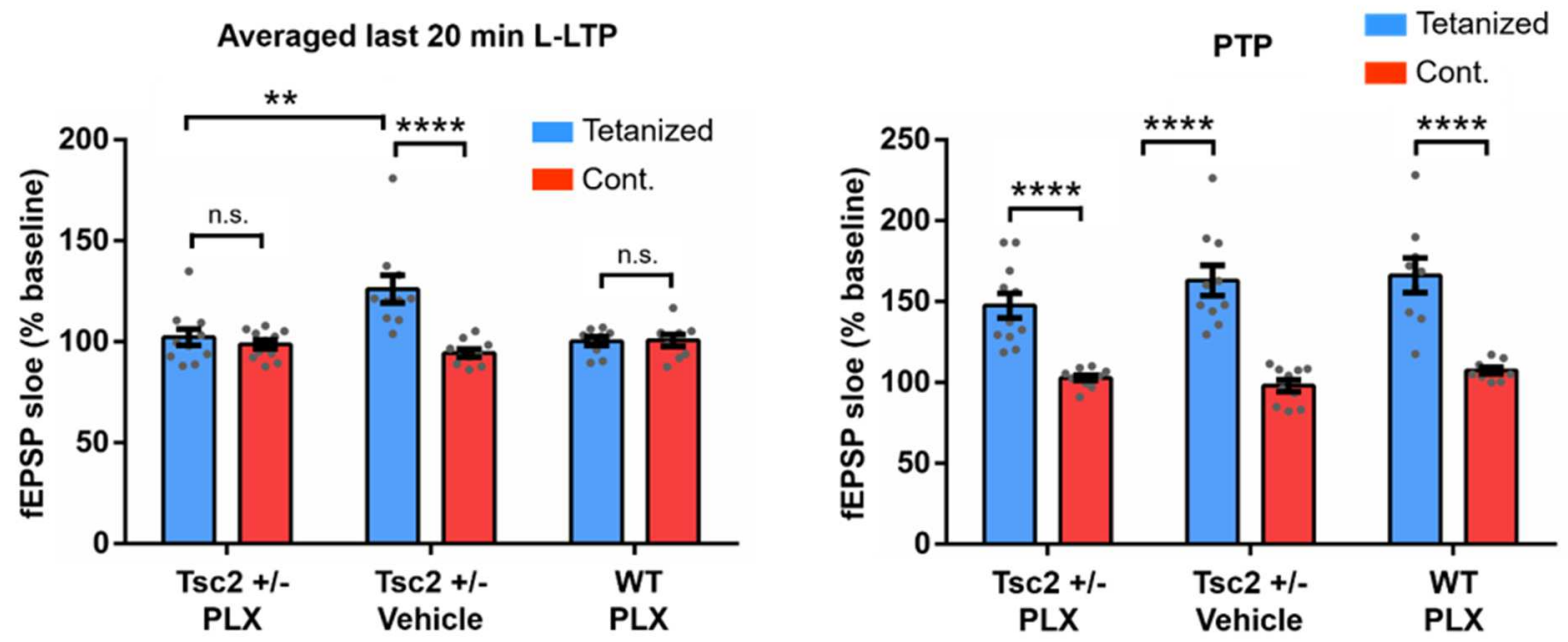
Fig. 5. Effects of microglia depletion in CA1 LTP of male Tsc2 ${ }^{+/-}$Ep mice.

(A) Outline of Poly I:C injections, treatment with PLX5622 and analysis approach. (B) Initial fEPSP slopes recorded in the CA1 region of hippocampal slices are shown before (baseline, 15 min) and following LTP induction (with a 1-s, 100-Hz tetanus delivered at time 0 ) for the tetanized pathway and for a separate, untetanized pathway (control). Data are plotted in 1-min blocks and fEPSP slopes are normalized to the average baseline response. Sample traces show responses (5 responses were averaged) during baseline (grey) and during the last $5 \mathrm{~min}$ of recordings (color). Scale bars, $1 \mathrm{mV}$ and $10 \mathrm{~ms}$. (C) Averaged fEPSPs during the last 20 min of LTP recording. Regular two-way ANOVA, pathway $\times$ genotype interaction: $F_{(2,54)}=10.51, p<0.001$; Sidak's multiple comparisons for tetanized vs. control pathway fEPSPs, $* * * * p<0.0001$ for Tsc2 ${ }^{+/-}$; VEH group, not significant (ns) for other two groups; Tukey's multiple comparisons for tetanized pathway fEPSPs, ** $p<0.01$ for Tsc $2^{+/-}$; PLX group vs. Tsc2 $2^{+/}$; VEH group. (D) Averaged posttetanus fEPSPs (5 min, PTP) recorded immediately after a 100-Hz tetanus. Regular two-way ANOVA, pathway factor $F_{(1,54)}=105.1, \mathrm{P}<0.001$, genotype factor $F_{(2,54)}=1.488, p>0.05$; Sidak's multiple comparisons for tetanized vs. control pathway, **** $p<0.0001$ for all three groups. Tsc $2^{+/-}$ ; VEH slices, $\mathrm{n}=10$ from 6 mice. Tsc $2^{+/-}$; PLX slices, $\mathrm{n}=11$ from 7 mice. WT; PLX slices, $\mathrm{n}=9$ slices from 5 mice. All data are shown as means \pm SEM. 


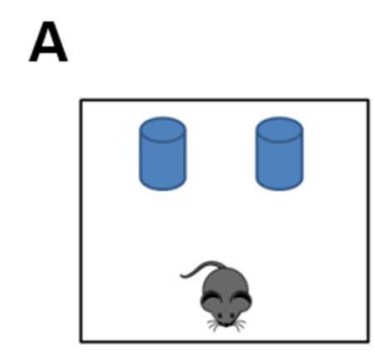

Training

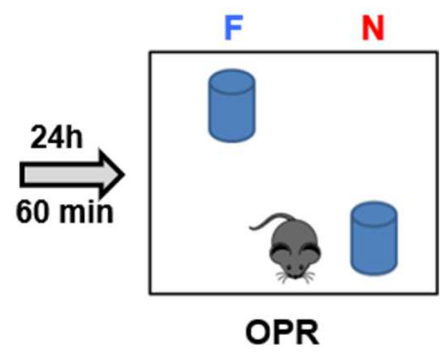

B

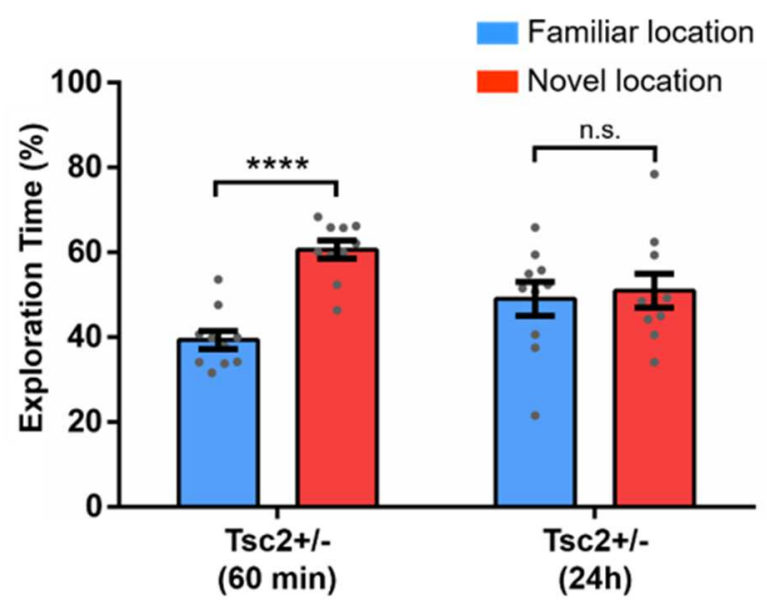

Fig. 6. Male Tsc2 ${ }^{+/-}$mice show normal OPR memory 60 min after training.

(A) Outline of behavior approach. (B) Graph shows the \% of time the mice spent actively exploring the objects in the novel and familiar locations. Tsc2 $2^{+/} / 60 \min (\mathrm{n}=10 ; \mathrm{P}<0.0001, \mathrm{t}=6.97)$ show normal OPR (they explore significantly more the object in the novel location than the object in the familiar location) but $\mathrm{Tsc}^{+/-} / 24 \mathrm{~h}(\mathrm{n}=10 ; \mathrm{P}=0.73, \mathrm{t}=0.33)$ mice show OPR deficits (show no preference for the object in the novel location). Data represent means \pm SEM as well as individual data. 


\section{A}

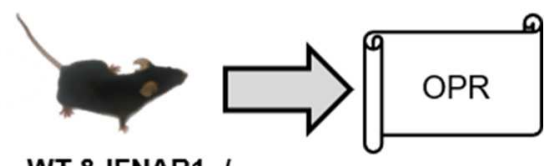

WT \& IFNAR1 $-/-$

WT \& Tsc2 +/-

B

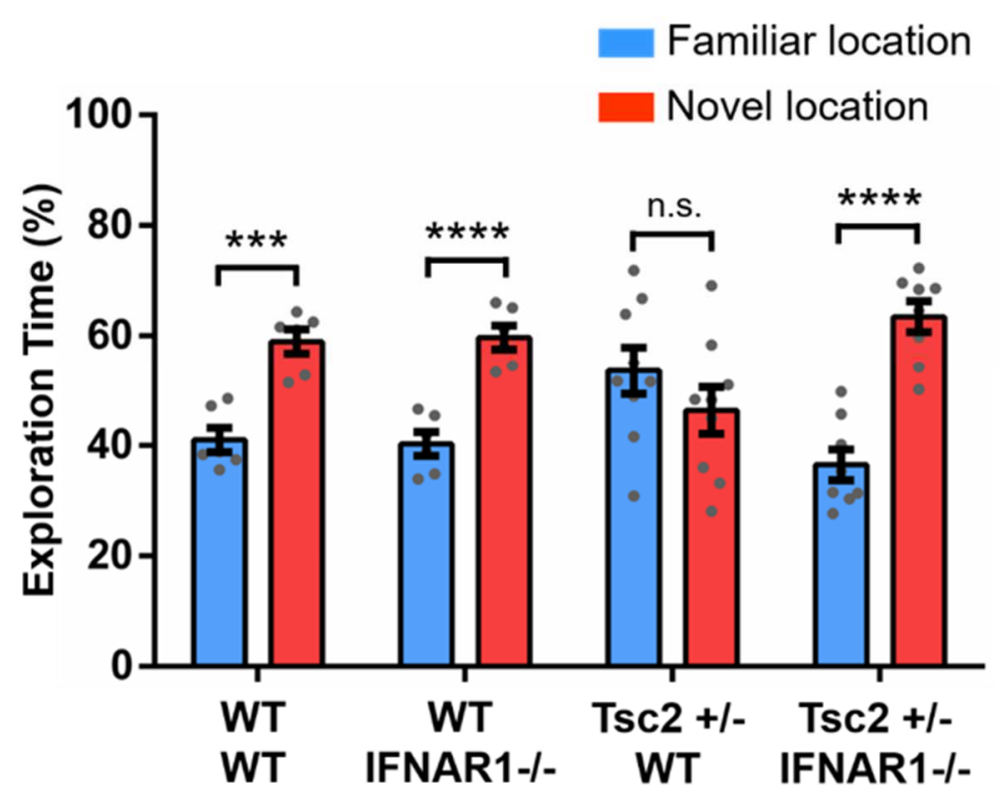

Fig. 7. Effects of the null homozygous mutation of type I IFN receptor (IFNAR1 ${ }^{-/-}$) in $\mathrm{Tsc}^{+/-}$ OPR deficits.

(A) Behavior approach. (B) $\mathrm{Tsc}^{+/-}(\mathrm{n}=9 ; \mathrm{P}=2.24, \mathrm{t}=1.19)$ show OPR deficits. WT ( $\mathrm{n}=6 ; \mathrm{P}<0.001$, $\mathrm{t}=5.71), \mathrm{IFNAR}^{-{ }^{--}}(\mathrm{n}=6 ; \mathrm{P}<0.0001, \mathrm{t}=6.34)$ and $\mathrm{Tsc}^{+/-} /$IFNAR1 $^{-/-}(\mathrm{n}=8 ; \mathrm{P}<0.0001, \mathrm{t}=6.78)$ show normal OPR. Data represent means \pm SEM as well as values for individual mice. 